\title{
The Application Value of Magnetic Resonance Imaging (MRI) in the Clinical Diagnosis in Hospital Management
}

\author{
Yan Chen ${ }^{1}$, Hong-Mei Zhou' ${ }^{2}$ Qi-Jie Jiang ${ }^{3}$
}

\begin{abstract}
Background \& Objective: To evaluate the application value of dynamic enhanced magnetic resonance imaging (DCE-MRI) in the clinical diagnosis in hospital based on the fuzzy C-means clustering algorithm and conduct its cost-benefit analysis in practice.

Methods: This study was conducted from October 11th 2020 to November 25, 2020. Eighteen patients with lung cancer confirmed by pathology were collected and examined by DCE-MRI one day before and one month after surgery using a fuzzy C-means clustering algorithm. And then, taking Guang'an District People's Hospital as an example, the present paper conducts the cost-benefit analysis with the utilization of net present value (NPV).

Results: After 1-month review, eleven patients with effective treatment, four numerical Ktrans, Kep, $\mathrm{Ve}, \mathrm{Vp}$ compared with preoperative decreased, which Ktrans, Kep, the change of the Ve was statistically significant $(P<0.05)$, the Vp of decline has no statistical significance $(P>0.05)$, the experimental and ineffective group after an operation, compared to four numerical Ktrans and Vp difference was statistically significant $(P<0.05)$, the corresponding AUC (area under the curve) to 1 and 0.818 , the sensitivity of $100 \%$ and $85.7 \%$, a specific degree of $100 \%$ and $72.7 \%$. The NPV analysis results showed that the hospital could achieve profit and loss balance in the third year after purchasing MRI equipment and then gradually obtain positive cash inflow.

Conclusion: The application of MRI in the Clinical Diagnosis helps hospital promote the therapeutic effect, thus having great practical value, and also contributes to increasing benefits in the long run.
\end{abstract}

KEYWORDS: Magnetic resonance imaging, Fuzzy C-means clustering algorithm, Curative effect, Clinical diagnosis, Hospital management.

Abbreviations: DCE-MRI: Dynamic enhanced magnetic resonance imaging. NPV: Net Present Value.

RECIST: Reliable Tumor Evaluation Criteria. CR: Complete Response.

doi: https://doi.org/10.12669/pjms.37.6-WIT.4791

How to cite this:

Chen Y, Zhou HM, Jiang QJ. The Application Value of Magnetic Resonance Imaging (MRI) in the Clinical Diagnosis in Hospital Management. Pak J Med Sci. 2021;37(6):1710-1713. doi: https://doi.org/10.12669/pjms.37.6-WIT.4791

This is an Open Access article distributed under the terms of the Creative Commons Attribution License (http://creativecommons.org/licenses/by/3.0), which permits unrestricted use, distribution, and reproduction in any medium, provided the original work is properly cited.

1. Yan Chen, Attending Doctor

Business School, Sichuan University, Chengdu, China.

2. Hong-Mei Zhou, Attending Doctor,

School of Public Administration,

Sichuan University, Chengdu, China.

3. Qi-Jie Jiang, Associate Professor

Business School, Chengdu University, Chengdu, China.

Correspondence:

Prof. Dr. Qi-Jie Jiang,

Business School, Chengdu University,

Chengdu, China.

Email: jiangqijie@cdu.edu.cn

* Received for Publication:

* Revision Received:

* Accepted for Publication:

April 5, 2021

May 16, 2021

June 30, 2021

\section{INTRODUCTION}

It is of great significance to carry out quality management on the laboratory and improve the quality level of the laboratory. Dynamic enhanced magnetic resonance imaging (DCE-MRI) is an emerging functional imaging technology in recent years. ${ }^{1-3}$ A series of parameters reflecting the hemodynamic changes of tumor micro-vessels are obtained through intravenous contrast agent injection and continuous multi-phase dynamic scanning. ${ }^{4}$ For example, at present, the incidence of lung cancer in China remains high. According 
Yan Chen et al.

Table-I: Paired sample T-test of preoperative and postoperative parameters in the effective group.

\begin{tabular}{lcccc}
\hline Time & Ktrans $/($ min -1$)$ & Kep $/(\min -1)$ & $V e$ & $V p$ \\
\hline preoperative & $0.321 \pm 0.235$ & $0.828 \pm 0.385$ & $0.205 \pm 0.108$ & $0.085 \pm 0.098$ \\
postoperative & $0.044 \pm 0.028$ & $0.348 \pm 0.155$ & $0.111 \pm 0.062$ & $0.079 \pm 0.108$ \\
$\mathrm{P}$ & 0.002 & 0.01 & 0.036 & 0.084 \\
\hline
\end{tabular}

to statistics, male lung cancer patients account for the first cause of cancer death, second only to breast cancer among women. Interventional therapy can be an effective treatment for patients who cannot accept surgery or are intolerant to chemo radiotherapy. However, some realistic problems need to be considered in the practice: can the hospital make a profit after purchasing the MRI equipment because the cost of MRI equipment is usually high? How long can it be profitable? This paper attempts to focus on these questions.

\section{METHODS}

Firstly, we used a 3.0T superconducting MAGNETIC resonance scanner (Discovery 750), eight-channel phased array coil, plus respiratory gating. All patients were subjected to a general MRI scan: axial T2PROPELLER (TR6300ms, TE75ms, layer thickness $5 \mathrm{~mm}$, layer spacing $1 \mathrm{~mm}$, FOV40 cm), Scanning time: $2 \mathrm{~min} 15 \mathrm{~s}$; DWI (B =800s/ $\mathrm{mm} 2$, TR5700ms, TE50ms, layer thickness $5 \mathrm{~mm}$, layer spacing $2 \mathrm{~mm}, F O V 40 \mathrm{~cm})$, Scan time $50 \mathrm{~S}$. Then scanned for DCE-MRI: first sweep 5 turn Angle of the Flip angle $3,6,9,12,15$, scanning sequence for BHAxLAVA - Flex - 3, 6, 9, 12, 15, scanning parameters for TR3. $6 \mathrm{~ms}$, TE1.7 ms, $5 \mathrm{~mm}$, with a thick layer of layer $1 \mathrm{~mm}$ spacing, FOV $40 \mathrm{~cm}$, each sequence scan time is $6 \mathrm{~s}$, then dynamic contrast enhancement scan sequence BHAxLAVA - Flex - 12 + C, scanning parameters for TR3.6 ms, TE1.7 ms, $5 \mathrm{~mm}$, with a thick layer of layer spacing of $1 \mathrm{~mm}$, FOV $40 \mathrm{~cm}$, before injection of contrast agent, the first phase was straightforward scanning, followed by high pressure injection of gadolinium diamine contrast agent $(0.1 \mathrm{mmol} / \mathrm{kg})$ at an injection rate of $2 \mathrm{ml} / \mathrm{s}$, and injection of $0.9 \%$ normal saline at the same rate of $20 \mathrm{ml}$, a total of 50 scanning periods, $6 \mathrm{~s}$ per phase, for a total of $5 \mathrm{~min}$. All patients were given routine therapy after surgery ${ }^{5}$ and reliable tumor evaluation criteria (RECIST) were used to evaluate the efficacy.

Secondly, we got five turn Angle and dynamic contrast-enhanced image import OK software. ROI was delineated at the same level for each patient before and after surgery, avoiding necrotic cystic changes and normal blood vessels, and four quantitative parameters were calculated: Ktrans (volume transfer constant), Kep(interstitial - plasma rate constant), Ve(extracellular space volume fraction), and $\mathrm{Vp}$ (plasma volume fraction). Finally, based on the annual cash flow of MRI, the cost of MRI is analyzed by calculating the net present value. ${ }^{6}$

\section{RESULTS}

After one month of treatment, the DCE-MRI images of 11 patients showed that the substantial part of the tumor's diameter decreased by more than $30 \%$, indicating that the treatment was effective. The seven patients belonged to SD or PD, that is, the invalid group and the change of the red area on the false-color map was not noticeable, or the range was more extensive than before. In the influential group, Ktrans, Kep, Ve, and Vp were all decreased compared with those before treatment, and the changes in Ktrans, Kep, and Ve were statistically significant $(\mathrm{P}=0.002, \mathrm{P}=0.01, \mathrm{P}=0.036)$, while the difference in $\mathrm{Vp}$ was not statistically significant $(\mathrm{P}=0.084)$. Postoperative Ktrans and $\mathrm{Vp}$ of the influential group and the non-effective group showed statistically significant differences $(\mathrm{P}=0.001, \mathrm{P}=0.036)$, corresponding AUC (area under the curve) were 1 and 0.818 , sensitivity $100 \%$ and $85.7 \%$, specificity $100 \%$, and $72.7 \%$, as shown in Table-I-III. Relevant ROC curves of Ktrans, Kep, $\mathrm{Ve}$, and $\mathrm{Vp}$ in all patients after surgery.

Table-II: Postoperative independent sample T-test of the effective group and the invalid group.

\begin{tabular}{lcccc}
\hline Grouping & Ktrans $/($ min- 1$)$ & Kep $/($ min- 1$)$ & $V e$ & $V p$ \\
\hline Effective group & $0.044 \pm 0.028$ & $0.348 \pm 0.155$ & $0.111 \pm 0.062$ & $0.079 \pm 0.108$ \\
The invalid group & $0.397 \pm 0.285$ & $0.519 \pm 0.323$ & $0.186 \pm 0.11$ & $0.311 \pm 0.312$ \\
$P$ & 0.001 & 0.148 & 0.081 & 0.036 \\
\hline
\end{tabular}

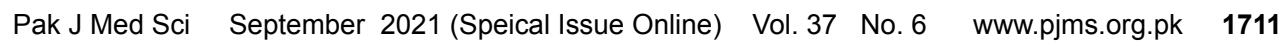


Table-III: AUC, maximum approximate index, sensitivity, and specificity of the influential group and the non-effective group after operation.

\begin{tabular}{lcccc}
\hline Statistical indicators & Ktrans & Kep & Ve & $V p$ \\
\hline AUC & 1 & 0.727 & 0.727 & 0.818 \\
Maximum approximation index & 1 & 0.584 & 0.494 & 0.584 \\
The sensitivity & 1 & 0.857 & 0.857 & 0.857 \\
Specific degrees & 1 & 0.727 & 0.636 & 0.727 \\
\hline
\end{tabular}

And then, taking Guang' an District People's Hospital as an exam ple, the present paper conducts the cost-benefit analysis with the utilization of net present value (NPV). The annual net present value of MRI is about two million RMB. The total investment income of MRI in 2001 and 2002 reached $75 \%$ of the investment cost of the equipment. In the third year, positive cash inflow can be realized. Through the analysis of 5 -year complete data of MRI in hospital by year, through the study of cost benefit development trend, it shows that with the operation of MRI, its economic benefits increase year by year. The NPV analysis results showed that the hospital could achieve profit and loss balance in the third year after purchasing MRI equipment and then gradually obtain positive cash inflow.

\section{DISCUSSION}

According to the two-compartment hemodynamic model proposed in the literature, ${ }^{7}$ tumor microcirculation characteristics, such as vascular size, distribution, permeability, ${ }^{8-10}$ etc. were evaluated using quantitative methods. Four parameters, Ktrans, Kep, Ve, and Vp, were obtained by fitting calculation.

The difference in Ktrans and Vp changes in the effective group and the ineffective group after treatment was significant. Among the four values, Ktrans had the highest sensitivity and specificity, indicating that Ktrans was used to evaluate tumor blood perfusion and predict the therapeutic effect, which was consistent with most scholars. ${ }^{11,12}$

The advantages of using dynamic contrastive enhanced MAGNETIC resonance quantitative parameters to evaluate the efficacy of lung cancer are as follows: first, morphological changes of the lesions and the supply of tumor microvessels can be observed at the same time to obtain rich diagnostic information, and the evaluation method is more accurate and objective. ${ }^{13}$ Second, this examination has no radiation effect and is more suitable for long-term follow-up reviews than $\mathrm{CT}$ and other examination methods. Third, the examination cost is moderate, easy to be accepted by patients. ${ }^{14}$ These results are consistence with several previous studies, for example, Zheng et al. ${ }^{15}$

\section{CONCLUSIONS}

DCE-MRI can effectively evaluate the curative effect of BACE treatment in lung cancer patients, which is objective, accurate, and non-invasive, and provides a new reference basis for clinical adjustment of the treatment plan. However, large sample data support and long-term follow-up indepth study are still needed. And the NPV analysis results showed that the hospital could achieve profit and loss balance in the third year after purchasing MRI equipment and then gradually obtain positive cash inflow and the net cash inflow in each year is increasing continuously.

Acknowledgement: We are immensely grateful to Guang'an District People's Hospital for its help in data collection.

Competing interests: None.

Funding: None.

\section{REFERENCES}

1. Fast MF, Eiben B, Menten MJ, Wetscherek A, Hawkes DJ, McClelland JR, et al. Tumour auto-contouring on $2 \mathrm{~d}$ cine MRI for locally advanced lung cancer: A comparative study. Radiother Oncol. 2017;125(3):485-491. doi: 10.1016/j. radonc.2017.09.013

2. Sheth KN, Mazurek MH, Yuen MM, Cahn BA, Shah JT, Ward A, et al. Assessment of brain injury using portable, low-field magnetic resonance imaging at the bedside of critically ill patients. JAMA Neurol. 2021;78(1):41-47. doi: 10.1001/jamaneurol.2020.3263.

3. Schroers M, Homsi R, Skowasch D, Buermann J, Zipfel M, Schild $\mathrm{HH}$, et al. Lung cancer screening with MRI: results of the first screening round. J Cancer Res Clin. 2018;144(1):117125. doi: $10.1007 /$ s00432-017-2521-4

4. Kuhl CK. Abbreviated magnetic resonance imaging (MRI) for breast cancer screening: rationale, concept, and transfer to clinical practice. Annu Rev Med. 2019;70:501-519. doi: 10.1146/annurev-med-121417-100403 
5. Cui L, Yin JB, Hu CH, Gong SC, Xu JF, Yang JS. Inter and intra observer agreement of ADC measurements of lung cancer in free breathing, breath-hold and respiratory triggered diffusion-weighted MRI. Clin Imag.2016;40(5):892-896. doi: 10.1016/j.clinimag.2016.04.002

6. Millon D, Byl D, Collard P, Cambier SE, Van Maanen AG, Vlassenbroek A, et al. Could new reconstruction CT techniques challenge MRI for the detection of brain metastases in the context of initial lung cancer staging. Eur Radiol. 2018;28(2):770-779. doi: 10.1007/s00330-017-5021-7

7. Chawla A, Carls G, Deng E, Tuttle E. The expected net present value of developing weight management drugs in the context of drug safety litigation. Pharmacoeconomics. 2015;33(7):749-763. doi: 10.1007/s40273-015-0300-0

8. Followill RA, Olsen BC. A closed-form, aftertax, net present value solution to the mortgage refinancing decision. Eng Econ.2015;60(3):165-182. doi: 10.1080/0013791X.2015.1014531

9. Wang H, Chandarana H, Block KT, Vahle T, Fenchel M, Das IJ. Dosimetric evaluation of synthetic CT for magnetic resonance-only based radiotherapy planning of lung cancer. Radiat Oncol. 2017;12(1):1-9. doi: 10.1186/s13014-017-0845-5

10. Manzoor J, Aftab S, Yaqoob M. Ambiguous genitalia: An overview of 7 years-experience at the Children's Hospital\& Institute of Child Health, Lahore, Pakistan. Pak J Med Sci.2019;35(1):151-155. doi: 10.12669/pjms.35.1.289

11. Bacchi S, Franke K, Wewegama D, Needham E, Patel S, Menon, D. Magnetic resonance imaging and positron emission tomography in anti-NMDA receptor encephalitis: A systematic review. J Clin Neurosci. 2018;52:54-59. doi:10.1016/j.jocn.2018.03.026
12. He Y, Wei L, Li W. Comparison of application values of CT and MRI in the diagnosis of early lacunar infarction. Pak J Med Sci. 2018;34(1):190-193. doi: 10.12669/pjms.341.13619

13. Puntmann VO, Carerj ML, Wieters I, Fahim M, Arendt C, Hoffmann J, et al. Outcomes of cardiovascular magnetic resonance imaging in patients recently recovered from coronavirus disease 2019 (COVID-19). JAMA Cardiol.2020;5(11):1265-1273. doi: 10.1001/ jamacardio.2020.3557

14. Junaid M, Bukhari SS, Saeed MA, Rashid MU. Mini titanium plates; hearkening the end of non-rigid cranial bone flap fixation. Pak J Med Sci. 2017;33(4):915-919. doi: 10.12669/ pjms.334.12003

15. Zheng W, Li B, Zou Y, Lou, F. The prenatal diagnosis and classification of cleft palate: The role and value of magnetic resonance imaging. Eur Radiol. 2019;29(10):5600-5606. doi:10.1007/s00330-019-06089-9

\section{Authors Contribution:}

YC: Conceived, designed and did statistical analysis \& editing of manuscript.

HMZ: Did data collection and manuscript writing. QJJ: Takes the responsibility and is accountable for all aspects of the work in ensuring that questions related to the accuracy or integrity of any part of the work are appropriately investigated and resolved. 